\title{
An Acoustic Time-frequency Approach to Condition Monitoring of Ventricular Assistive Devices
}

\author{
Ian R. Prechtt ${ }^{1}$, Steven W. Day ${ }^{2}$, and Jason R Kolodziej ${ }^{3}$ \\ 1,2,3 Rochester Institute of Technology, Rochester, NY, 14623, USA \\ irp2474@rit.edu \\ swdeme@rit.edu \\ jrkeme@rit.edu
}

\begin{abstract}
This paper studies the acoustic signals of left ventricular assist devices (LVADs) as it relates to machine health. Current LVAD condition monitoring requires examination from trained medical professionals, and is both inefficient and roughly-prognostic. To better quantify a patient's condition, the diagnostic method must be robust, non-invasive, and simple to apply. The concept behind this work is to determine an identifying pattern between the specific acoustics produced by an LVAD with the related overall health of the patient. Due to the cycle-to-cycle variance of heart sounds, the continuous wavelet transform (CWT) is applied to the objective audio signal so that a high resolution spectra is obtained. From this, region specific image features are developed and subsequently used in a support vector machine (SVM) algorithm to classify between health conditions. The preliminary goal is to develop an accurate and non-invasive diagnostic method for determining patient health that can be applied for any LVAD variant. This process is validated through in vitro testing using a DC motor as an LVAD proxy.
\end{abstract}

\section{INTRODUCTION}

Implant graft stenosis (narrowing) and localized thrombosis (clotting), among other failure modes, have limited the long term viability of left ventricular assistive devices (LVADs), as alternatives to orthotopic surgery. LVADs are motors implanted from the left ventricle to aorta that assist in blood circulation of patients who suffer from heart disease and related illnesses. With current one year survival rates already nearing that of surgery (Slaughter et al., 2007) there is strong potential for LVADs to replace surgery as the long term alternative, however doing so requires mitigation of the related failure modes and issues of the devices.

Ian Prechtl et al. This is an open-access article distributed under the terms of the Creative Commons Attribution 3.0 United States License, which permits unrestricted use, distribution, and reproduction in any medium, provided the original author and source are credited.
Current diagnostics and prognostics of ventricular assistive devices is difficult given the range of devices, patients, condition severities, and technological limitations. Currently, the most practical diagnosis requires a combination of several indicators and tests administered by trained medical professionals. It has been well established and proven that thoracic sounds are definitive indicators of abnormal health condition (Slaughter et al., 2007). Extrapolating upon this finding, several other authors have shown that this holds true for LVAD/artificial heart patients as well (Masson \& Rieu, 1998) (Granegger et al., 2006). Furthermore, there have been several successful attempts at using artificial intelligence (Makino et al., 2005) (Kim et al., 1995) and classifiers (Loghmanpour et al., 2015) to automatical identify related abnormalities/conditions.

A commonality among those that use machine learning routines is that the routine features are frequency spectrum (Yost, Royston, Bhat, \& Tatooles, 2016) (Kim et al., 1995) or differentially (Wang \& Simaan, 2013) determined. More advanced techniques have been applied to thoracic sounds (Nogata et al., 2012) (Tang, Li, Park, \& Qiu, 2010) however not when assistive devices are considered. This paper will examine the viability of using time-frequency spectra image recognition of LVAD acoustics to discern condition for the intent of issue mitigation.

\section{BIOLOGiCAl SySTEM}

Accurately modeling the physicalities and characteristics of the thoracic environment, in an in vitro setup, is required for proper LVAD analysis. While simulating the local thoracic acoustics (cardiac, pulmonary, etc.) is impractical, we are able to achieve the physiological LVAD boundary conditions of left ventricular pressure, aortic pressure, and pulsatile flow/phase. This is done using the system shown in Figure 1.

The system detailed is a single side heart phantom, specifically the left side, as that is the side of LVAD grafting. We generate pulsatile flow within the system from a compressible 


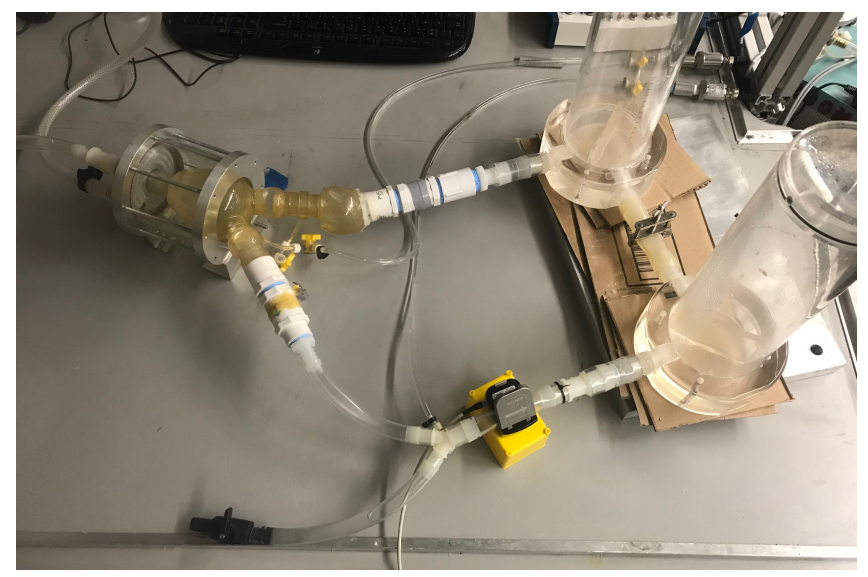

Figure 1. Circulatory physiology simulator.

actuating polymer that mimics the left ventricle. From this component we also have control over the ventricular pressure of the system which is actuated through air compression. The specific timing of the pulse is triggered via an external function generation. Unidirectional flow of the system is maintain through biological heart valve phantoms. Both the mitral and aortic valves (left chamber valves) are mimicked using passive bileaflet valves. They provide low resistance throughflow, and minimal phase delay when closing. Just as with the left chamber, when in systole (cardiac contraction) the aortic bileaflet valve opens while the mitral bileaflet closes allowing the ventricle surface to "push" its retained fluid to the aorta. When in diastole (cardiac relaxation), the contrary occurs, allowing the ventricle to "pull" fluid in, to then be released in subsequent systole (cardiac compression).

The final motor boundary condition, aortic pressure, is achieved through a series of retaining chambers. Flow between these chambers is controlled manually via a clamp (resistance component) for the purpose of tuning the system to the desired boundary conditions, and reducing bileaflet valve delay. Whereas the pulsatile flow and ventricular pressure conditions are controllable parameters, the aortic pressure and specific magnitude of the flow are reliant on the chamber to chamber resistance. In this way, the chamber-clampchamber series acts as a capacitive-resistive-capacitive circuit, where the retained chamber fluid is indicative of system capacitance. The first chamber (immediately after the aortic valve, and before the clamp) is the aortic component, while the latter (after the clamp, before the mitral valve) is the atrial component. The atrial chamber is vented to ambient, and so maintaining low atrial pressures is elementary, aside from the fact that atrial pressure is not a direct boundary condition of the potential assistive device. The aortic chamber however does not vent for the purpose of mimicking biological compliance.

$$
C=\Delta V / \Delta P
$$

Compliance, $\mathrm{C}$, is the quantitative measurement of the elasticity of biological arteries and veins. Depending on the stage of the cardiac cycle, arteries and veins dilate and contract in response to pressure and flow changes. Compliance itself is an influencing factor in patients with hyper/hypotension which merits incorporation into this work. Compliance is the quotient of Volume, V, and Pressure, P, meaning that compliance linearly scales with compressibility. Using this relationship, we are able to implement a compliance factor through an air pocket in the aortic chamber. When in systole, the fluid pushes against the pocket, compressing it, mimicking circulatory dilation. Similarly, while in diastole the pressurized pocket then expands, mimicking circulatory contraction. The magnitude to which this occurs is too a direct result of the resistance of the retaining chamber series, thus while not an additional independent control surface it is sufficient.

Performance of the system can be quantified solely in terms of the aortic pressure, assuming flow is correctly correlated. Peak ventricular pressure strongly matches systolic pressure (in non LVAD patients), while the diastolic pressure is regulated via the retaining chamber series elements. These trends are most applicable under no fault scenario as the load on the assistive device is minimal, however the system's behavior becomes increasingly more nonlinear as the device load increases. We approximately judge the fidelity of the system through comparison to the idealized response of the Wiggers diagram (model of physiological left chamber blood pressures). The system can be tuned to accurately mimic healthy cardiac operation (Figure 2), and so by then alternatively tuning the system we can then simulate both abnormal conditions (hypotension and hypertension) requiring assistive device implementation and healthy variation. Similarly, while the assistive device is functional in the system loop the performance can then be appropriately tuned to mimic the actual physiological state that the device produces.

\subsection{Assistive Device}

For preliminary testing an Anself 12VCD brushless pump was used in lieu of an LVAD (Noted that a HeartMate 2 is procured for follow up trials) for several reasons, most notably potential motor health effects. In order to classify between various health conditions the motor/graft is faulted several ways. It was not known if this would harm the LVAD or to what degree, and so we used a cheaper product as a replacement. Both function in essentially the same manner (neglecting control scheme) and so we can directly extrapolate our findings from one device to the other. It is also more practical in these initial trials to have control of device performance, as the motor is manually controlled via VDC power source. Select assistive devices such as the HeartMate 3 produce non-constant flow rates due to intentional pulsating, which in and of itself is a failure mitigation protocol. Aside from the fact that an LVAD's control module 
may innately interfere with the fidelity of the fault testing any variations in pump performance will manifest in the related frequency spectrum. While normally not a significant issue, physiological heart sounds are non-stationary. "Smearing" of the individual spectra components would increase cross-term components, proving analysis much more difficult. Similarly, by having complete control over the pump allows for testing over a more consistent and comprehensive range. For this testing the motor is driven with a 6VDC signal, at 7800 revolutions per minute.

In previous work (Yost et al., 2016) a silicone based molding, EcoFlex 00-10, is used to facilitate thoracic acoustic transmission between an assistive device and a stethoscope diaphragm. The same material is used for this work, however with the purpose of vibration dampening rather than transmission. The motor is set in a $5.5 \times 4 \times 3.25$ " block of the molding, reducing the motor's vibrational output to the base of the simulator. The silicone molding was not de-aired for this testing.

\subsection{Seeded-Fault Conditions}

In this initial work only one fault condition is considered, outlet graft occlusion. An occlusion blockage is positioned downstream of the pump, prior to the aorta. This fault is characterized by a $75 \%$ decrease in the graft cross sectional area. The physical blockage is contained within a removable component of the faulting piece, see Figure 3. This allows for interchangeable faults into the system. When operating nominally (no occlusion) we implement a $0 \%$ occlusion component. Future trials will consider another severity of fault (50\% blockage) along with upstream (inlet graft) blockages. Assuming the signal indicators of the fault manifest more

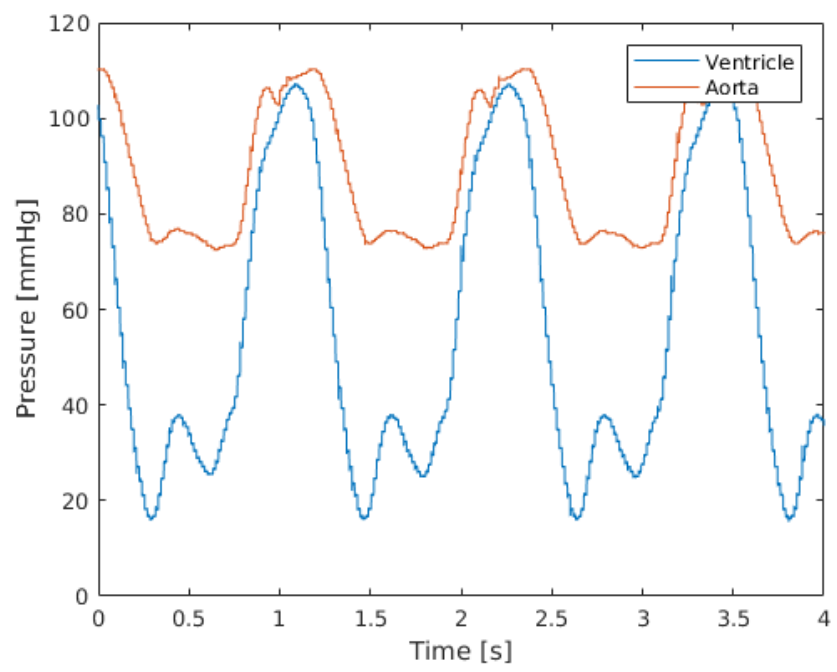

Figure 2. Physiological simulator health pressure curves mimicked to the Wiggers model of ideal biological left chamber pressures.

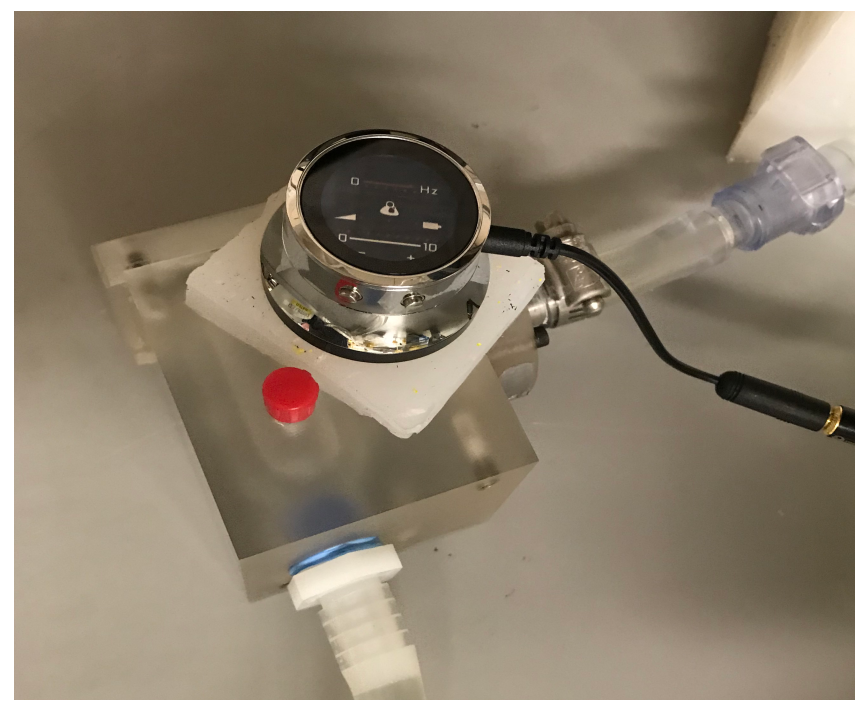

Figure 3. Blockage fault component with stethoscope positioned on the listening surface.

prominently with increasing fault severity, the $75 \%$ occlusion is used to potentially elicit the most apparent results.

The blockage component is machined to have several flat instrumentation surfaces. For this work the only surface utilized was the top-most so that no direct holding pressure is necessary (Figure 3). The occlusion element used is indicative of a generalized instance of outlet blockage (increased resistance, geometrical alteration, etc.) however in a patient this blockage would be more localized to the LVAD outlet stator.

\subsection{Data Acquisition}

In recent years a wide range of digital stethoscopes have become available for listening to heart sounds. Both commercial stethoscopes (Yost et al., 2016) and custom devices (Song, Jia, Lu, \& Tao, 2012) have been used for similar purposes. For this work, a ThinkLabs Digital One is used (Figure 3). The Digital One utilizes a capacitive sensor instead of a piezoelectric sensor to record heart sounds. For the test iterations the stethoscope is sampled at $10 \mathrm{kHz}$ and is operated in single channel wideband $(20 \mathrm{~Hz}$ to $2000 \mathrm{~Hz})$ streaming to an National Instruments USB-6212 module. The stethoscope diagram is placed atop a $1.3 \mathrm{~cm}$ thick square of EcoFlex at the top-most surface of the fault component to mimic thoracic acoustic transmission. In order to sync periodic cycles, the trigger signal of ventricular compression and decompression is also recorded by the data acquistion module. This signal is indicative of an EKG signal, which is assumedly readily available in a medical environment, and offers the highest fidelity cycle syncing potential. For all iterations a trigger frequency of $.85 \mathrm{hz}$ ( 50 beats per minute) is used to regulated phase.

Two TDH40 pressure transducers are used to monitor ven- 
tricular and aortic pressures. Monitoring these parameters enables control of the assistive device boundary conditions, which allows us to meet various biological conditions. Flow, while only monitored for verification, is attained using a Transonic flow meter. These three parameters are used solely for monitoring and recording the simulator states, i.e. they are not directly used in a processing algorithm.

\section{Acoustic Signal Processing}

Biological heart sounds are non-stationary signals that can vary in both characteristics (murmurs) and frequency (rate). The trigger frequency of the simulator can be globally fixed. Because of system compliance the pressure boundary conditions are not guaranteed to be globally consistent, and so the system does carry innate variation. Similarly, as one would expect, the sound and flow produced by the motor varies across cycles. As such the simulator audio can be postprocessed in the same manner as physiological signals.

Using the stethoscope, a ten second audio sample is collected. Using an auto-peak recognition algorithm on the trigger signal the individual cycles of the file are parsed, creating the ensemble set. The individual cycles are then aligned and subsequently averaged. Averaging the ensemble reduces the variation of the individual cycles, while preserving significant components. As is present in the shown ensemble of Figure 4, three distinguishable components are preserved, while significantly decreasing the adjacent variation. Maintaining these peaks, indicators of both systolic diastolic phase, are crucial for further identifying potentially significant intervals of device operation.

$$
x(t)_{\text {ensemble }}=\operatorname{mean}\left\{x(t)_{i}\right\}
$$

Due to the utilization of the trigger signal the cycle set, $x(t)_{i}$, is always consistently synced. As asserted before the simulator does not show the same physiological variation as a biological circulatory system and so given a fixed trigger signal frequency, the resulting cycles will be of the same period, or near same due to decimation. If this were not the case then the data can be linearly/nonlinearly scaled to an assumed period.

\subsection{Time-Frequency Analysis}

Biological heart sounds are non-stationary, or are characterized by non-noise cycle-to-cycle variations. The specific timing of frequency varying events then becomes more important to identify. For example the physical opening and closing of heart valves are directly responsible for generating specific low and high frequency spectrum content (Wood \& Barry, 1995). Thus if we know at which timed frequency bands to look, we can then identify physiological events and/or conditions.

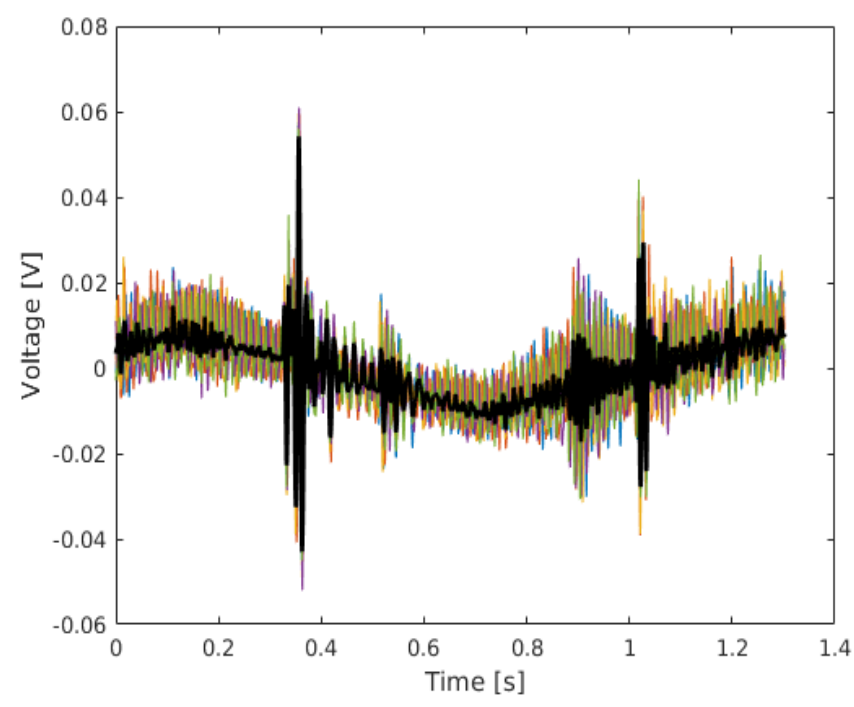

Figure 4. Ensembled average of unfaulted audio cycles (bold). Ensemble is indicated by the background cycles.

We apply the Morlet basis continuous wavelet transform (CWT) to the ensemble averaged acoustic signal to develop the time-frequency spectra of the audio signal. The time scale of the spectra is then normalized to a [0 1] space for a consistent reference frame. Doing so in theory allows for valid comparison between ensembles of various frequencies, however for this preliminary work such is not examined.

The faulted spectra shows to have both a greater intensity on average (Figure 5) and a slightly different phase response as compared to the unfaulted data. This however is only obvious when comparing against the same intensity scaling which is not practical for untested states/conditions. Instead the maximum and minimum values of the intensity matrix are used to develop individual scalings, and we instead look for regions where the 'shape' of the spectra is the predominant indicator of health. We term this subspace of the spectra the region of interest (ROI).

The greatest signal intensity occurs about the three peaks preserved by the ensemble. When normalized this occurrence is not apparent without the individual scalings and so we instead look inbetween the peaks (S1-S2 cardiac beat). This correlates to a frequency bound of $\left[2^{4}, 2^{8}\right] \mathrm{hz}$ and a normalized time bound of $[.4, .6]$. Even when intensity normalized there is a clear change in the spectra when faulted (Figure 6). The motor frequency $(128 \mathrm{hz})$ is more or less consistent in this region, however the occlusion blockage increases the motor load which manifests as various frequency components in the ROI band.

From the ROI subspace we then convert each intensity matrix to both a discrete 256 level grayscale image and a minimized variance binary image (Figure 6). This enables us to develop image specific features of each ROI. From the grayscale im- 

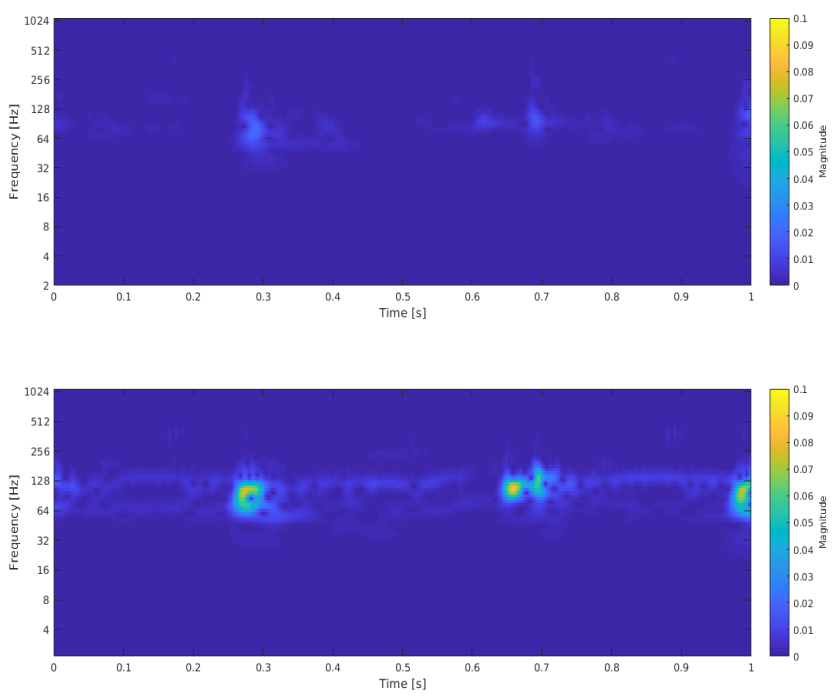

Figure 5. Consistent scaling unfaulted spectra (Top). Consistent scaling $75 \%$ occulsion faulted spectra (Bottom)

age we develop a set of 13 texture features. Similarly, we derive 18 shape features from the binary image. All 31 features are scaled by their mean and deviation for a more consistent classification basis, as is typical in classification algorithms.

\section{Classification and Results}

Classification between the faulted and unfaulted states is accomplished using a support vector machine (SVM), using the 31 image features as the classifier input. The SVM, while binarily classifying between two condition classes, is trained from a range of pressure states. These states are defined by both the systolic (maximum aortic pressure) and dias-

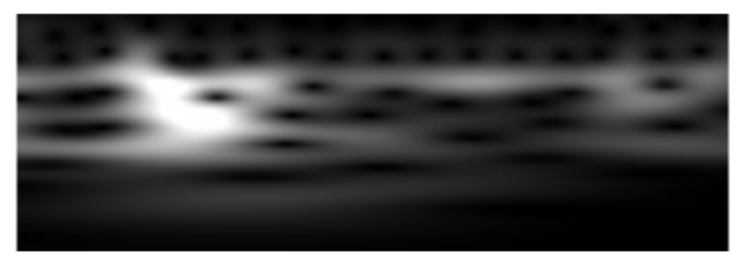

(a)

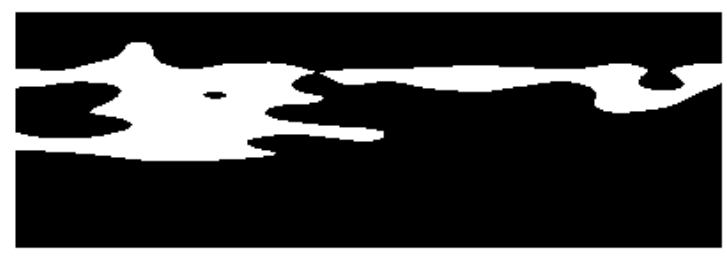

(b) tolic (minimum aortic pressure) ensemble pressures. This is extremely beneficial for establishing comparable training and testing regions, and for also evaluating the practicality of those states/regions - specifically in terms of hypotension (low blood pressure) and hypertension (high blood pressure).

Under the assumption that hypotension and hypertension are in and of themselves potential indicators of health, the primary training and testing state region is the area between a systolic pressure of $130-90 \mathrm{mmHg}$ and a diastolic pressure of 80-60 mmHg. These boundaries are outlined by the dashed lines of Figure 7. The training and testing states are limited to this region in order to correlate to physiological healthy conditions of LVAD operation within a patient, supporting the fidelity of the acoustic signals produced.

Nevertheless several states mildly outside of this region were used for increased variation, and validation purposes. A set of 137 unique states are used in training the SVM, of which 69 were unfaulted. Of these states approximately $23 \%$ are indicative of very mild hypotension or hypertension. The testing states are compiled similarly, with $28 \%$ of the total 25 states indicating the same issues. Additionally, the majority of both the training and testing states are localized along the peak pressure $(130 / 80 \mathrm{mmHg})$ to minimum pressure $(90 / 60$ $\mathrm{mmHg}$ ) diagonal of the region.

Classifier performance is quantified in three metrics, overall correct classifications, correct healthy classifications (positive predictive value - PPV) and correct faulted classifications (negative predictive value - NPV).

The classifier generally performs more accurately on the faulted states than on the unfaulted states (ignoring instances when testing with the training space). Specifically in terms

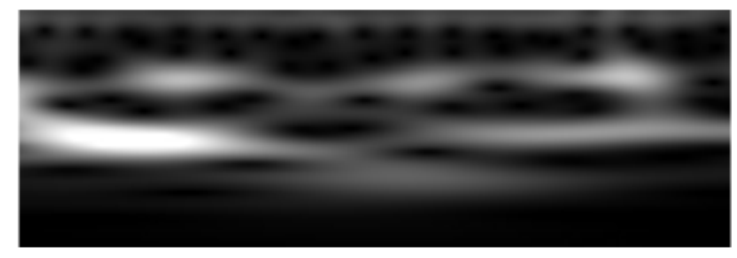

(c)

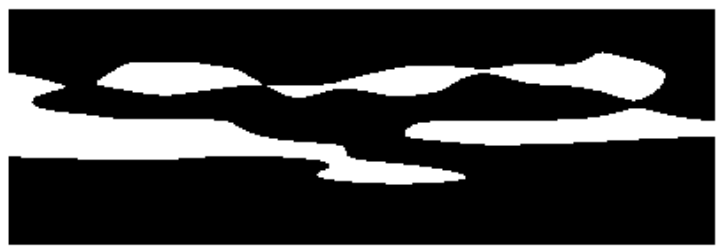

(d)

Figure 6. ROI derived images. (a): Unfaulted ROI grayscle image. (b): Unfaulted ROI binary image. (c): Faulted ROI grayscale image. (d): Faulted ROI binary image. 

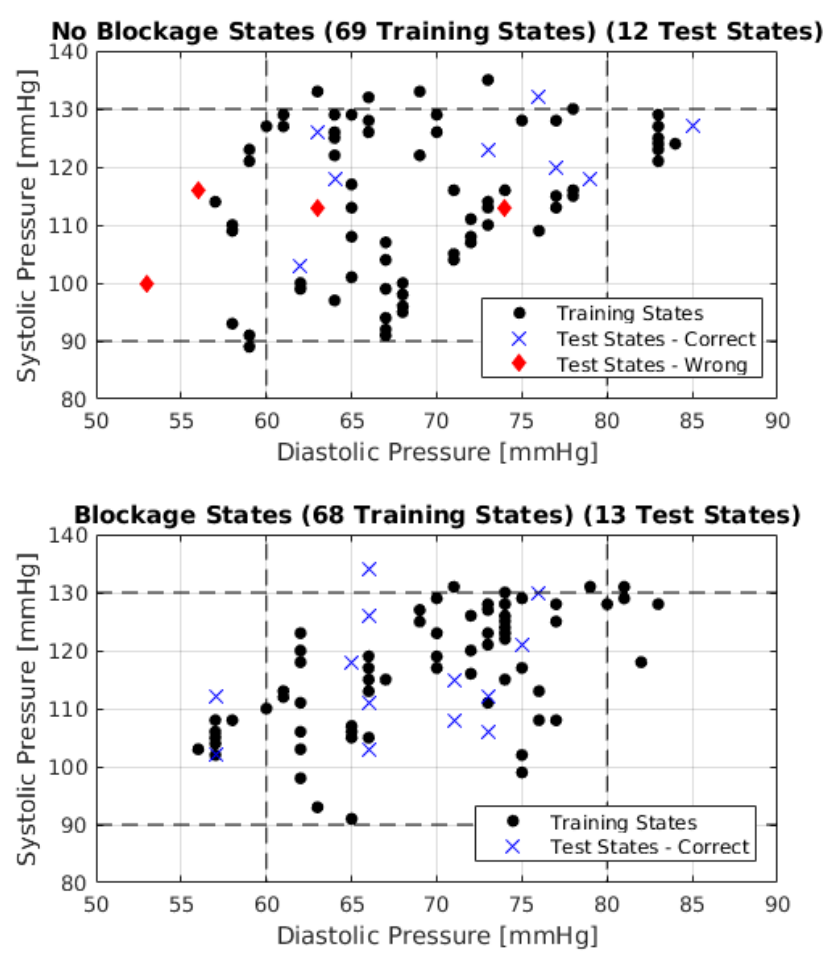

Figure 7. Gaussian kernel SVM Results for no occlusion states (top) and 75\% occulsion states (bottom)

of the Gaussian kernel model (Figure 7), all faulted states are correctly identified, even the outliers of the region. The unfaulted states however have a much higher degree of misidentification. The unfaulted region is much less dense than that of the faulted states and so we theorize that this significantly impacts the misidentifications of the unfaulted region. Outside of that area by large the validation states have few to no adjacent training states, which confirms that expectation that these states are the most susceptible to classification error.

Among the kernels trialed the polynomial (seventh order) kernel performs best Table 1 . This finding however only holds for when using all 31 image features as classifier states. When using either solely the grayscale texture features or the binary shape features the Gaussian kernel performs most consistently.

Table 1. Classification success rates when using an independent validation set (Training-Testing) or when using the training set as the validation set (Training-Training)

\begin{tabular}{|l|c|c|c|}
\cline { 2 - 4 } \multicolumn{1}{c|}{} & \multicolumn{3}{c|}{ Classifier Score [\%] } \\
\hline $\begin{array}{c}\text { Classifier Test } \\
\text { Set }\end{array}$ & $\begin{array}{c}\text { Linear } \\
\text { Kernel }\end{array}$ & $\begin{array}{c}\text { Gaussian } \\
\text { Kernel }\end{array}$ & $\begin{array}{c}\text { Polynomial Kernel } \\
\text { (7th order) }\end{array}$ \\
\hline Testing & 76.00 & 84.00 & 92.00 \\
\hline Testing (PPV) & 58.33 & 66.67 & 84.61 \\
\hline Testing (NPV) & 85.71 & 100.00 & 85.71 \\
\hline Training & 80.29 & 92.70 & 100.00 \\
\hline Training (PPV) & 81.16 & 95.65 & 100.00 \\
\hline Training (NPV) & 79.41 & 89.71 & 100.00 \\
\hline
\end{tabular}

\section{Conclusion}

Both the Gaussian and seventh order polynomial kernel SVMs correctly identified the validation states at rates upwards of $80 \%$. In the specific cases of misidentifications a consistent trend among all models is that there is a believed inverse relationship between the potential for misidentification of a specific testing state and the density of that test state's adjacent training points. A comprehensive solution to this would be to simply increase the quantity of training states relative to test states.

Even when utilizing a small number of training states the classifier does perform "better" in terms of the identification of faulted states as compared to unfaulted states. Medically speaking, if this holds true even when trained with a more comprehensive state set this is the less problematic scenario, comparing against the opposite, to indicate a false health concern when healthy, rather than to indicate health when that is not true.

Additionally, with the feasibility of using the spectra "shape", rather than intensity, being confirmed we can expect that this method would directly apply to a range of other fault severities and potential other fault types. If we consider additional fault types we may not necessary see the manifestations in the elected ROI, however by augmenting with additional ROIs or features we would expect to achieve similar results.

\section{REFERENCES}

Granegger, M., Masetti, M., Laohasurayodhim, R., Schloeglhofer, T., Zimpfer, D., Schima, H., \& Moscato, F. (2006). Continuous monitoring of aortic valve opening in rotary blood pump patients. In Ieee transactions on biomedical engineering.

Kim, H., Park, S., Hindrichs, H., Bearson, G., Khanwilkar, P., \& Olsen, D. (1995). Noninvasive diagnosis of mechanical failure of the implanted total artificial heart using neural network analysis of acoustic signals. ASAIO, 41(M271-M276), 360-364.

Loghmanpour, N., Kanwar, M., Druzdzel, M., Benza, R., Murali, S., \& Antaki, J. (2015). A new bayesian network-based risk stratification model for prediction of short-term and long-term lvad mortality. ASAIO, 61(3), 313-323.

Makino, T., Okamoto, E., Tanaka, S., Yasuda, T., Inoue, Y., Saito, I., .. Mitamura, Y. (2005). Estimation of early-state malfunction using implantable artificial heart sound in animal experiments. Artifical Organs, 30(5), 360-364.

Masson, J., \& Rieu, R. (1998). Time-frequency analysis of the noise produced by the closing of artificial heart valves: an in vitro study. Medical Engineering \& Physics, 20(1), 418-431. 
Nogata, F., Yokota, Y., Kawanura, Y., Morita, H., Uno, Y., \& Walsh, W. (2012). Audio-visual recognition of auscultatory heart sounds with fourier and wavelet analyses. In Transactions on biomedical engineering and image recognition.

Slaughter, M., Feldman, C., Sobieski, M., Silver, M., Martin, M., Kurien, S., ... Pederson, B. (2007). Utilization of acoustic signatures to identify heartmate xve device end-of-life. Journal of Heart and Lung Transplantation, 26(5), 579-583.

Song, D., Jia, L., Lu, Y., \& Tao, L. (2012). Heart sounds monitor and analysis in noisy environments. In International conference of systems and informatics.

Tang, H., Li, T., Park, Y., \& Qiu, T. (2010). Seperation of heart sound signal from noise in joint cycle frequencytime-frequency domains based on fuzzy detection. In Ieee transactions on biomedical engineering.

Wang, Y., \& Simaan, M. (2013). A suction detection system for rotary blood pumps based on the lagrangian support vector machine algorithm. IEEE Journal of Biomedical and Health Informatics, 17(3), 654-663.

Wood, J., \& Barry, D. (1995). Time-frequency analysis of the first heart sound. IEEE Engineering in Medicine and Biology, 14(2), 144-151.

Yost, G., Royston, T., Bhat, G., \& Tatooles, A. (2016). Acoustic characterization of axial flow left ventricular assist device operation in vitro and in vivo. ASAIO, 62(6), 46-55. 